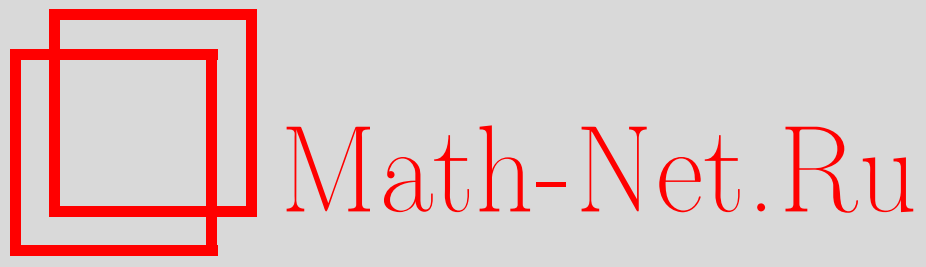

С. А. Степин, Спектр резонансов и формула следа в задаче потенциального рассеяния, Функи. анализ и его прил., 2004, том 38, выпуск 3, 79-89

DOI: https://doi.org/10.4213/faa119

Использование Общероссийского математического портала MathNet.Ru подразумевает, что вы прочитали и согласны с пользовательским соглашением

http://www . mathnet.ru/rus/agreement

Параметры загрузки:

IP : 3.91 .87 .62

26 апреля 2023 г., 13:29:13

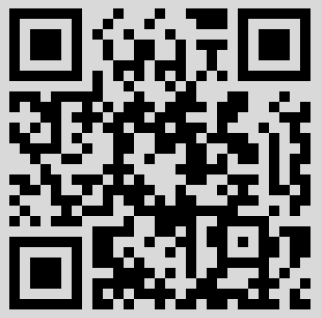


Функииональный анализ и его приложения

2004, т. 38 , вып. 3, с. 79-89

УДК 517.9

\title{
Спектр резонансов и формула следа в задаче потенциального рассеяния*
}

\author{
(C) 2004. С. А. Степин
}

\author{
Виктору Борисовичу Лидскому \\ с благодарностью и глубоким уважением
}

В настоящей статье асимптотическое распределение резонансов (квазиуровней) оператора Шрёдингера исследуется в рамках спектрального подхода Лакса и Филлипса к изучению решений волнового уравнения

$$
\left(\frac{\partial^{2}}{\partial t^{2}}-\Delta+V(x)\right) u(x, t)=0
$$

с вещественным потенциалом $V(x) \in C_{0}^{\infty}\left(\mathbb{R}^{n}\right)$ в пространстве нечетной размерности. Как известно, связь между возмущенной и невозмущенной $(V(x) \equiv 0)$ эволюционными группами $U_{V}(t)$ и $U_{0}(t)$, отвечающими задаче Коши для уравнения (1), задается волновыми операторами и оператором рассеяния; последний в спектральном представлении невозмущенной задачи записывается с помощью матрицы рассеяния.

Согласно общей теории [1], решение $u(x, t)$ уравнения (1) имеет при больших $t$ асимптотическое представление вида

$$
u(x, t) \sim \sum_{\lambda \in \Lambda} c(\lambda) e^{i \lambda t} w_{\lambda}(x),
$$

где $\Lambda \subset \mathbb{C}-$ множество полюсов аналитического продолжения матрицы рассеяния, а $w_{\lambda}(x), \lambda \in \Lambda$, - так называемые резонансные состояния, являющиеся решениями уравнения Шрёдингера

$$
\left(-\Delta+V-\lambda^{2}\right) w=0 .
$$

Если $\operatorname{Im} \lambda<0$, то $w_{\lambda} \in L^{2}\left(\mathbb{R}^{n}\right)$ и, стало быть, $\lambda^{2}-$ собственное значение оператора $-\Delta+V$. В случае, когда потенциал $V(x)$ - вещественная финитная функция, все собственные значения - отрицательные и потому $\Lambda \cap \mathbb{C}_{-} \subset i \mathbb{R}_{-}$. Если же $\operatorname{Im} \lambda \geqslant 0$, то резонансное состояние $w_{\lambda} \notin L^{2}\left(\mathbb{R}^{n}\right)$ выделяется условием (cм. [2])

$$
w_{\lambda}(x)=\frac{e^{-i \lambda r}}{r^{(n-1) / 2}} f(x, \lambda),
$$

где $f(x, \lambda)$ в окрестности бесконечности представляется рядом по обратным степеням $r=|x|$ с коэффициентами, зависящими от $\theta=x / r$ и $\lambda$.

*Работа выполнена в рамках проекта, поддержанного грантом Минпромнауки России МД-251.2003.01. 
В таком контексте возникает вопрос о локализации и асимптотическом распределении резонансных частот $\lambda \in \Lambda$. В общем случае (т.е. для потенциалов $V \in C_{0}^{\infty}\left(\mathbb{R}^{n}\right)$ общего положения) известны (см. [3]) лишь оценки сверху для соответствующей считающей функции; их вывод основан на трактовке резонансных частот как особенностей аналитического продолжения резольвенты соответствующего оператора Шрёдингера (см. об этом в §1). Более трудной является задача получения нижней оценки асимптотического распределения резонансов. Для этой цели эффективным средством оказывается рассмотрение множества $i \Lambda$ как спектра генератора полугруппы Лакса-Филлипса. Явное описание этого генератора (см. §2) в так называемом трансляционном представлении получено в теореме 1, которая дополняет и развивает отмеченную в [1] аналогию с уравнением переноса.

Далее, в $\S 3$ и 4 настоящей работы показано, как можно использовать формулу регуляризованного следа (см., например, [3])

$$
\operatorname{Tr}\left(U_{V}(t)-U_{0}(t)\right)=\sum_{\lambda \in \Lambda} e^{i \lambda t}, \quad t>0,
$$

для исследования структуры множества $\Lambda$. Эта формула получается с помощью теоремы Лидского [4] о совпадении матричного и спектрального следов и позволяет судить о возможной степени разреженности членов последовательности $\left\{\lambda_{n}\right\}$ по поведению суммы ряда Дирихле $\sum e^{i \lambda_{n} t}$ при $t \downarrow 0$ (теорема 2). Именно теоремы 1 и 2 (вместе со следствием о локализации резонансов) составляют основные результаты статьи.

Для простоты изложения будет рассматриваться случай, когда оператор Шрёдингера $-\Delta+V$ в $L^{2}\left(\mathbb{R}^{n}\right)$ не имеет собственных значений. Это предположение не является ограничительным с точки зрения вопроса об асимптотическом распределении точек $\lambda \in \Lambda$, поскольку их количество в нижней полуплоскости конечно, если $V \in C_{0}^{\infty}\left(\mathbb{R}^{n}\right)$.

Различным аспектам обсуждаемой здесь проблематики и смежным вопросам посвящены статьи и монографии [5-8]. Результаты недавних исследований в этой области отражены в [9] и [10]. Как стало известно автору из отзыва рецензента, анализ асимптотических свойств резонансов на основе связи с генератором полугруппы Лакса-Филлипса проводился в 60-е годы в группе Б. С. Павлова.

Обзор литературы по рассматриваемому предмету будет дан в статье автора, которая готовится к публикации.

\section{$\S 1$. Особенности аналитического продолжения резольвенты оператора $-\Delta+V$}

При сделанных выше предположениях для произвольных $\omega \in \mathbb{S}^{n-1}$ и $\lambda \in$ $\mathbb{R} \backslash\{0\}$ существует единственное решение уравнения (2) со следующей асимптотикой по пространственной переменной:

$$
e^{i \lambda x \cdot \omega}+\frac{e^{-i \lambda r}}{r^{(n-1) / 2}}\left(a(\theta, \omega, \lambda)+O\left(\frac{1}{r}\right)\right),
$$

где $x \cdot \omega-$ скалярное произведение, $r=|x|$ и $\theta=x / r \in \mathbb{S}^{n-1}$. Коэффициент $a(\theta, \omega, \lambda)$ представляет собой предельную амплитуду рассеяния плоской волны, двигающейся в направлении $\omega$ и отраженной рассеивателем (потенциалом $V$ ) 
в направлении $\theta$. Известно, что амплитуда рассеяния $a(\theta, \omega, \lambda)$ гладко зависит от $\theta$ и $\omega$, аналитична по $\lambda$ в нижней полуплоскости и продолжается в верхнюю полуплоскость как мероморфная функция.

Матрицей рассеяния принято называть семейство интегральных операторов $S_{V}(\lambda)$ в пространстве $L^{2}\left(\mathbb{S}^{n-1}\right)$ с ядрами

$$
\delta(\theta-\omega)+\left(\frac{i \lambda}{2 \pi}\right)^{(n-1) / 2} a(-\theta, \omega, \lambda) .
$$

Описание множества резонансов как множества полюсов оператор-функции $S_{V}(\lambda)$ основано на исследовании аналитических свойств амплитуды рассеяния $a(\theta, \omega, \lambda)$ (см. [11]).

Другое описание множества $\Lambda$ базируется на использовании резольвентного тождества

$$
R_{V}(\lambda)=\left(-\Delta+V-\lambda^{2}\right)^{-1}=R_{0}(\lambda)\left(I+V R_{0}(\lambda)\right)^{-1} .
$$

Здесь $R_{0}(\lambda)$ - интегральный оператор, ядро которого есть функция Грина для уравнения Гельмгольца. Если шар $\Omega=\{|x| \leqslant d\}$ содержит носитель потенциала $V$, то отображения $V R_{0}(\lambda): L^{2}(\Omega) \rightarrow L^{2}(\Omega)$ образуют аналитическое в $\mathbb{C}$ семейство компактных операторов и, согласно аналитической теореме Фредгольма, $\left(I+V R_{0}(\lambda)\right)^{-1}$ продолжается в верхнюю полуплоскость как мероморфная оператор-функция, имеющая вычеты конечного ранга. В соответствии с этим принято говорить об аналитическом (мероморфном) продолжении резольвенты $R_{V}(\lambda)$, причем справедливо следующее (см. [2] и [3])

ПРЕДЛОЖЕНИЕ 1. Множество $\Lambda$ состоит из тех значений параметра $\lambda \in \mathbb{C}$, для которых оператор $I+V R_{0}(\lambda)$ необратим в $L^{2}(\Omega)$.

Резонансу $\lambda \in \Lambda$ отвечает нетривиальное решение $w_{\lambda} \in C^{\infty}\left(\mathbb{R}^{n}\right)$ уравнения $(2)$, имеющее на бесконечности определенную асимптотику. При этом

$$
w_{\lambda}(x)=\frac{e^{-i \lambda r}}{r^{(n-1) / 2}}\left(h(\theta)+O\left(\frac{1}{r}\right)\right), \quad r=|x| \rightarrow \infty,
$$

и резонансное состояние $w_{\lambda}(x)$ связано с непрерывной функцией $h(\theta)$ формулой

$$
w_{\lambda}(x)=\left(\frac{-i \lambda}{2 \pi}\right)^{(n-1) / 2} \int_{\mathbb{S}^{n-1}} h(\omega)\left(e^{i \lambda x \cdot \omega}+\phi(x, \omega, \lambda)\right) d \omega,
$$

где $\phi(x, \omega, \lambda)=-R_{V}(-\lambda) V e^{i \lambda x \cdot \omega}-$ решение уравнения

$$
\left(-\Delta+V(x)-\lambda^{2}\right)\left(e^{i \lambda x \cdot \omega}+\phi(x, \omega, \lambda)\right)=0 .
$$

Приведенное выше описание множества $\Lambda$ используется (см. [10]) при получении оценки сверху для считающей функции количества резонансов. Вывод указанной оценки основан на применении формулы Йенсена к определителю $\operatorname{Det}\left(I-\left(V R_{0}(\lambda)\right)^{(n+1) / 2}\right)$, являющемуся целой функцией, множество нулей которой содержит $\Lambda$.

ЗАмЕчАниЕ. С точки зрения функциональной модели Сёкефальви-Надя и Фояша матрица рассеяния $S_{V}(\lambda)$ представляет собой характеристическую функцию генератора полугруппы Лакса-Филлипса, а описанный выше подход к оценке (сверху) количества резонансов, по-существу, совпадает с известным 
в этой теории приемом построения скалярного кратного характеристической функции (см., например, [8]) в виде подходящего детерминанта Фредгольма.

\section{§2. Рассеяние в представлении Лакса-Филлипса}

Как было отмечено во введении, с задачей Коши для волнового уравнения (1) стандартным образом связано семейство операторов $U_{V}(t)$, задающих эволюцию начальных данных и образующих однопараметрическую группу. Обозначим через $\mathscr{H}$ конечно-энергетическое пространство, являющееся пополнением множества гладких начальных данных $\left\{u_{0}(x), u_{1}(x)\right\}$ с компактными носителями по норме

$$
\left\{\int_{\mathbb{R}^{n}}\left(\left|\nabla u_{0}\right|^{2}+\left|u_{1}\right|^{2}\right) d x\right\}^{1 / 2}
$$

Оператор $\left(\begin{array}{cc}0 & I \\ \Delta-V & 0\end{array}\right)$, определенный в $\mathscr{H}$ в слабом смысле (см. [1]), служит генератором ограниченной группы $U_{V}(t)$ в $\mathscr{H}$; через $U_{0}(t)$ обозначается унитарная группа в $\mathscr{H}$, отвечающая случаю $V \equiv 0$.

Матрица рассеяния $S_{V}(\lambda)$, введенная выше (см. 1$)$, возникает здесь при

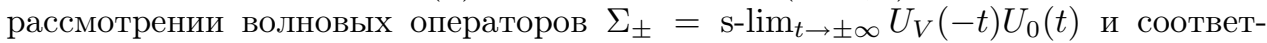
ствующего оператора рассеяния $\Sigma_{+}^{-1} \Sigma_{-}$, связывающего в терминах невозмущенной задачи асимптотики решений уравнения (1) при $t \rightarrow+\infty$ и $t \rightarrow-\infty$. А именно, оператор рассеяния действует как умножение на оператор-функцию $S_{V}(\lambda): L^{2}\left(\mathbb{S}^{n-1}\right) \rightarrow L^{2}\left(\mathbb{S}^{n-1}\right)$ в спектральном представлении группы $U_{0}(t)$.

$\mathrm{C}$ последним связано (посредством преобразования Фурье) так называемое трансляционное представление. Переход в него реализует изометрический изоморфизм $\mathscr{R}$ пространств $\mathscr{H}$ и $L^{2}\left(\mathbb{R} \times \mathbb{S}^{n-1}\right)$, переводящий $U_{0}(t)$ в группу сдвигов

$$
W_{0}(t)=\mathscr{R} U_{0}(t) \mathscr{R}^{-1}: \varphi(s, \omega) \mapsto \varphi(s-t, \omega) .
$$

Переход в трансляционное представление для гладких $u_{0}$ и $u_{1}$ осуществляется по формуле (см. [1])

$$
\mathscr{R}\left(\begin{array}{l}
u_{0} \\
u_{1}
\end{array}\right)=\frac{1}{2}(2 \pi)^{(1-n) / 2}\left(\partial_{s}^{(n+1) / 2}\left(R u_{0}\right)(s, \omega)-\partial_{s}^{(n-1) / 2}\left(R u_{1}\right)(s, \omega)\right),
$$

где $\partial_{s}=\partial / \partial s$ и $R$ - преобразование Радона,

$$
R u(s, \omega)=\int_{x \cdot \omega=s} u(x) d \mu=\int_{\mathbb{R}^{n}} u(x) \delta(s-x \cdot \omega) d x .
$$

Следуя схеме Лакса и Филлипса, введем подпространства

$$
\mathscr{D}_{ \pm}=\left\{f \in \mathscr{H}: U_{V}( \pm t) f(x)=0,|x|<t+d, t>0\right\}
$$

и обозначим через $\mathscr{P}_{+}\left(\mathscr{P}_{-}\right)$ортопроекторы в $\mathscr{H}$ на $\mathscr{D}_{+}^{\perp}$ (соответственно на $\left.\mathscr{D}_{-}^{\perp}\right)$. Семейство операторов $\mathscr{P}_{+} U_{V}(t) \mathscr{P}_{-}$при $t \geqslant 0$ образует полугруппу, отображающую подпространство $\left(\mathscr{D}_{+} \oplus \mathscr{D}_{-}\right)^{\perp}$ в себя. Отметим, что $\mathscr{R} D_{+}=$ $L^{2}\left((d, \infty) \times \mathbb{S}^{n-1}\right)$ и $\mathscr{R} D_{-}=L^{2}\left((-\infty,-d) \times \mathbb{S}^{n-1}\right)$ и, стало быть, $\mathscr{R}\left(\left(\mathscr{D}_{+} \oplus \mathscr{D}_{-}\right)^{\perp}\right)=$ $L^{2}\left((-d, d) \times \mathbb{S}^{n-1}\right)$. Ограничение на это инвариантное подпространство операторов указанной полугруппы, записанных в трансляционном представлении, обозначим через $Z_{V}(t)$. Согласно [3], справедливо следующее 
УтвеРЖДЕниЕ 1. Генератор $L_{V}$ полугруппъ Лакса-Филлипса $Z_{V}(t)$ имеет дискретный спектр $\sigma\left(L_{V}\right)=i \Lambda$, причем кратности собственных значений оператора $L_{V}$ совпадают с кратностями соответствующих полюсов оператор-функции $\left(I+V R_{0}(\lambda)\right)^{-1}$.

Явное описание генератора полугруппы $Z_{V}(t)$ дает

Tеорема 1. Действующий в пространстве $\mathscr{K}=L^{2}\left((-d, d) \times \mathbb{S}^{n-1}\right)$ оператор $L_{V}$ на своей области определения $D\left(L_{V}\right)=\left\{\varphi \in \mathscr{K}: \partial_{s} \varphi \in \mathscr{K}, \varphi(-d, \omega)=0\right\}$ имеет вид $L_{V}=-\partial_{s}+A$, где $A-$ ограниченный оператор на плотном в $\mathscr{K}$ множестве, определенный формулой

$$
A \varphi(s, \omega)=\frac{(-1)^{(n+1) / 2}}{2(2 \pi)^{n-1}} \partial_{s}^{(n-1) / 2} \int_{x \cdot \omega=s} V(x) \int_{\mathbb{S}^{n-1}}\left(\partial_{s}^{(n-3) / 2} \varphi\right)(x \cdot \theta, \theta) d \theta d \mu .
$$

ДокАЗАтЕльство. Введем обозначение $W_{V}(t):=\mathscr{R} U_{V}(t) \mathscr{R}^{-1}$. Тогда, как нетрудно видеть,

$$
Z_{V}(t)=\left.\mathscr{P}_{\mathscr{K}} W_{V}(t)\right|_{\mathscr{K}}, \quad t \geqslant 0,
$$

где $\mathscr{P}_{\mathscr{K}}$ - ортопроектор на $\mathscr{K}$ в $L^{2}\left(\mathbb{R} \times \mathbb{S}^{n-1}\right)$. Наряду с $Z_{V}(t)$ рассмотрим полугруппу

$$
Z_{0}(t)=\left.\mathscr{P}_{\mathscr{K}} W_{0}(t)\right|_{\mathscr{K}}, \quad t \geqslant 0,
$$

и ее генератор $L_{0}$, резольвента которого в полуплоскости $\operatorname{Re} \lambda>0$ действует на элемент $\varphi \in \mathscr{K}$ по формуле

$$
\left(L_{0}-\lambda I\right)^{-1} \varphi(s, \omega)=-\int_{0}^{\infty} e^{-\lambda t} Z_{0}(t) \varphi(s, \omega) d t=-\int_{-d}^{s} e^{\lambda(\tau-s)} \varphi(\tau, \omega) d \tau .
$$

Отсюда непосредственно следует, что генератор полугруппы $Z_{0}(t)$ есть оператор $L_{0}=-\partial_{s}$, определенный на функциях, обращающихся в нуль в точке $s=-d$.

Для векторов $\varphi \in L^{2}\left(\mathbb{R} \times \mathbb{S}^{n-1}\right)$, таких, что $\partial_{s} \varphi \in L^{2}\left(\mathbb{R} \times \mathbb{S}^{n-1}\right)$, существует предел

$$
\lim _{t \downarrow 0} \frac{\left(W_{V}(t)-W_{0}(t)\right) \varphi}{t}=\mathscr{R}\left(\begin{array}{cc}
0 & 0 \\
V & 0
\end{array}\right) \mathscr{R}^{-1} \varphi
$$

причем оператор $B=\mathscr{R}\left(\begin{array}{cc}0 & 0 \\ V & 0\end{array}\right) \mathscr{R}^{-1}$ ограничен в $L^{2}\left(\mathbb{R} \times \mathbb{S}^{n-1}\right)$ и, стало быть, генератор полугруппы $Z_{V}(t)$ имеет вид

$$
L_{V}=-\partial_{s}+A, \quad \text { где } A=\left.\mathscr{P}_{\mathscr{K}} B\right|_{\mathscr{K}} .
$$

Воспользуемся формулой обращения преобразования Радона

$$
u(x)=\frac{1}{2}(2 \pi)^{-n+1} \int_{\mathbb{S}^{n-1}}\left(\left(i \partial_{s}\right)^{n-1} R u\right)(x \cdot \omega, \omega) d \omega
$$

для вычисления действия оператора $B$ на плотном в $L^{2}\left(\mathbb{R} \times \mathbb{S}^{n-1}\right)$ множестве $\mathscr{R}\left(C_{0}^{\infty}\left(\mathbb{R}^{n}\right) \times C_{0}^{\infty}\left(\mathbb{R}^{n}\right)\right) \subset C_{0}^{\infty}\left(\mathbb{R} \times \mathbb{S}^{n-1}\right)$. Положим $\varphi=\mathscr{R}\left(\begin{array}{l}u_{0} \\ u_{1}\end{array}\right)$, где $u_{0}, u_{1} \in$ $C_{0}^{\infty}\left(\mathbb{R}^{n}\right)$. В силу свойства «четности» преобразования Радона, $R u(s, \omega)=$ $R u(-s,-\omega)$, имеем

$$
\left(\partial_{s}^{(n+1) / 2} R u_{0}\right)(s, \omega)=(2 \pi)^{(n-1) / 2}\left(\varphi(s, \omega)+(-1)^{(n+1) / 2} \varphi(-s,-\omega)\right),
$$


и, согласно формуле обращения,

$$
u_{0}(x)=(-2 \pi)^{-(n-1) / 2} \int_{\mathbb{S}^{n-1}}\left(\partial_{s}^{(n-3) / 2} \varphi\right)(x \cdot \theta, \theta) d \theta .
$$

Таким образом,

$$
B \varphi(s, \omega)=-\frac{1}{2}(2 \pi)^{-(n-1) / 2} \partial_{s}^{(n-1) / 2} R\left(V u_{0}\right)(s, \omega),
$$

где $u_{0}(x)$ задается формулой (4). Как видно непосредственно из определения преобразования Радона, $R u(s, \omega)=0$ при $|s|>d$, если носитель функции $u(x)$ принадлежит шару $\Omega=\{|x| \leqslant d\}$. Поскольку $\operatorname{supp} V \subset \Omega$, то $\operatorname{Ran} B \subset \mathscr{K}$ и, стало быть, $A$ есть сужение оператора $B$ на подпространство $\mathscr{K}=L^{2}((-d, d) \times$ $\left.\mathbb{S}^{n-1}\right)$.

ЗАмечАниЕ. Явный вид оператора $L_{V}$ указывает на его сходство с генератором эволюционной полугруппы, возникающей в задаче о переносе частиц, описываемом линеаризованным уравнением Больцмана (см. [12]). Аналогия между интегральными уравнениями для экспоненциальных мод в случае волнового уравнения и в случае уравнения переноса отмечалась в [1].

\section{§3. Конструкция параметрикса по Адамару и формула следа}

Известно (см., например, [13]), что для произвольной функции $\rho(t) \in C_{0}^{\infty}(\mathbb{R})$ оператор $\int_{\mathbb{R}}\left(U_{V}(t)-U_{0}(t)\right) \rho(t) d t: \mathscr{H} \rightarrow \mathscr{H}$ ядерный. Функционал

$$
\operatorname{Tr} \int_{\mathbb{R}}\left(U_{V}(t)-U_{0}(t)\right) \rho(t) d t
$$

задает обобщенную функцию $\Psi_{V}(t)=\operatorname{Tr}\left(U_{V}(t)-U_{0}(t)\right)$, носитель сингулярной части которой сосредоточен в нуле. При этом справедлива следующая (см. [3])

ЛЕмма 1. Имеет место разложение

$$
\Psi_{V}(t)=\sum_{j=1}^{(n-1) / 2} c_{j}(V) \partial_{t}^{n-1-2 j} \delta(t)+\Phi_{V}(t),
$$

где $\Phi_{V}(t)$ - регулярная обобщенная функиия, представимая своим рядом Тейлора

$$
\Phi_{V}(t)=|t|^{-n} \sum_{(n+1) / 2}^{\infty} c_{j}(V) t^{2 j}
$$

коэффициенты $c_{j}(V)$ получаются в результате интегрирования определенных многочленов от потенциала $V(x)$ и его производных, которые вычисляются рекуррентно.

Представление (5)-(6) выводится из формулы

$$
\operatorname{Tr}_{\mathscr{H}}\left(U_{V}(t)-U_{0}(t)\right)=2 \operatorname{Tr}_{L^{2}\left(\mathbb{R}^{n}\right)}(\cos (t \sqrt{-\Delta+V})-\cos (t \sqrt{-\Delta})) ;
$$

обобщенное ядро $K_{V}(t, x, y)$ оператора $\cos (t \sqrt{-\Delta+V})$ есть решение задачи

$$
\begin{gathered}
\left(\partial_{t}^{2}-\Delta+V(x)\right) K_{V}(t, x, y)=0 \\
K_{V}(0, x, y)=\delta(x-y), \quad \partial_{t} K_{V}(0, x, y)=0 .
\end{gathered}
$$


Поскольку $\int_{\mathbb{R}}\left(K_{V}(t, x, y)-K_{0}(t, x, y)\right) \rho(t) d t-$ непрерывное ядро, то

$$
\operatorname{Tr}_{\mathscr{H}}\left(U_{V}(t)-U_{0}(t)\right)=2 \int_{\mathbb{R}^{n}}\left(K_{V}(t, x, x)-K_{0}(t, x, x)\right) d x .
$$

Конструкция параметрикса по Адамару (см., например, [14]) применительно к волновому оператору $\partial_{t}^{2}-\Delta+V$ позволяет (ср. [15]) выписать разложение

$$
K_{V}(t, x, x)=\sum_{j=0}^{(n-1) / 2} \kappa_{j}(x) \partial_{t}^{n-1-2 j} \delta(t)+\sum_{(n+1) / 2}^{\infty} \kappa_{j}(x)|t|^{2 j-n},
$$

коэффициенты $\kappa_{j}(x)$ которого с точностью до постоянных множителей (зависящих от $j$ ) совпадают с $v_{j}(0, x)$, где

$$
v_{j}(\xi, \eta)=\int_{0}^{1}\left(\left(\Delta_{\xi} v_{j-1}\right)(t \xi, \eta)-V(t \xi+\eta) v_{j-1}(t \xi, \eta)\right) d t, \quad v_{0}(\xi, \eta) \equiv 1
$$

Отсюда видно, что $\kappa_{j}(x)$ оказываются многочленами от потенциала $V(x)$ и его производных. В случае $n=3$ имеем

$$
\kappa_{0}(x)=-\frac{1}{2 \pi}, \quad \kappa_{1}(x)=-\frac{1}{4 \pi} V(x), \quad \kappa_{2}(x)=\frac{1}{16 \pi}\left(-\frac{1}{6} \Delta V(x)+\frac{1}{2} V^{2}(x)\right),
$$

и, таким образом, при $t \downarrow 0$

$$
\Psi_{V}(t)=-\frac{\delta(t)}{2 \pi} \int V d x+\frac{t}{16 \pi} \int V^{2} d x+O\left(t^{3}\right) .
$$

Получить для регуляризованного следа $\operatorname{Tr}\left(U_{V}(t)-U_{0}(t)\right)$ «спектральное» представление в терминах резонансов (т.е. разложение по экспонентам с резонансными частотами) позволяет

ЛЕмма 2. Для $\rho(t) \in C_{0}^{\infty}\left(\mathbb{R}_{+}\right)$справедливо равенство

$$
\operatorname{Tr} \int_{\mathbb{R}_{+}}\left(U_{V}(t)-U_{0}(t)\right) \rho(t) d t=\operatorname{Tr} \int_{\mathbb{R}_{+}}\left(Z_{V}(t)-Z_{0}(t)\right) \rho(t) d t .
$$

ДоКАЗАТЕЛЬСТво подобного утверждения в случае рассеяния на препятствии дано в [13]. Применительно к случаю потенциального рассеяния заметим, прежде всего, что группа $U_{V}(t)$ унитарна относительно скалярного произведения, порожденного формой

$$
\int_{\mathbb{R}^{n}}\left(\left|\nabla u_{0}\right|^{2}+\left|u_{1}\right|^{2}+V(x)\left|u_{0}\right|^{2}\right) d x .
$$

Вследствие этого и ввиду включений $U_{V}(t) \mathscr{D}_{-} \subset \mathscr{D}_{-}$при $t<0$ и $\operatorname{supp} V \subset$ $\{|x| \leqslant d\}$ для произвольных $f \in \mathscr{D}_{-}$и $h \in \mathscr{H}$ имеем

$$
\left(f, U_{V}(t) h\right)_{\mathscr{H}}=\left(U_{V}(-t) f, h\right)_{\mathscr{H}}, \quad t>0 .
$$

Как известно, $U_{V}(t)=U_{0}(t)$ на подпространствах $\mathscr{D}_{+}$и $\mathscr{D}_{-}$для положительных и отрицательных $t$ соответственно. Таким образом, $\mathscr{D}_{+} \subset \operatorname{Ker}\left(U_{V}(t)-U_{0}(t)\right)$, $t>0$, и для произвольных $f \in \mathscr{D}_{-}$и $h \in \mathscr{H}$

$$
\left(f,\left(U_{V}(t)-U_{0}(t)\right) h\right)_{\mathscr{H}}=\left(\left(U_{V}(-t)-U_{0}(-t)\right) f, h\right)_{\mathscr{H}}=0, \quad t>0,
$$


т. е. $\operatorname{Ran}\left(U_{V}(t)-U_{0}(t)\right) \subset \mathscr{D}_{-}^{\perp}$. Поэтому при $t>0$

$$
U_{V}(t)-U_{0}(t)=\mathscr{P}_{-}\left(U_{V}(t)-U_{0}(t) \mathscr{P}_{+},\right.
$$

где $\mathscr{P}_{ \pm}-$ортопроекторы в $\mathscr{H}$ на $\mathscr{D}_{ \pm}^{\perp}($ см. $\S 2)$. Далее, учитывая равенство $\mathscr{P}_{K}=\mathscr{R} \mathscr{P}_{+} \mathscr{P}_{-} \mathscr{R}^{-1}$, согласно $(3)$, получаем

$$
\begin{aligned}
\operatorname{Tr} \int_{0}^{\infty} & \left(U_{V}(t)-U_{0}(t)\right) \rho(t) d t=\operatorname{Tr} \int_{0}^{\infty} \mathscr{P}_{-}\left(U_{V}(t)-U_{0}(t)\right) \mathscr{P}_{+} \rho(t) d t \\
\quad & \operatorname{Tr} \int_{0}^{\infty} \mathscr{P}_{+}\left(U_{V}(t)-U_{0}(t)\right) \mathscr{P}_{-} \rho(t) d t=\operatorname{Tr} \int_{0}^{\infty}\left(Z_{V}(t)-Z_{0}(t)\right) \rho(t) d t .
\end{aligned}
$$

СЛЕДСТВИЕ. Если $\operatorname{supp} \rho(t) \subset(2 d, \infty)$, mo $Z_{0}(t) \rho(t)=0 u$

$$
\operatorname{Tr} \int_{\mathbb{R}_{+}}\left(U_{V}(t)-U_{0}(t)\right) \rho(t) d t=\sum_{\lambda_{k} \in \Lambda} \int_{\mathbb{R}_{+}} e^{i \lambda_{k} t} \rho(t) d t .
$$

В работе [16] для произвольного фиксированного $\tau>0$ в пространстве $\mathscr{K}=L^{2}\left((-d, d) \times \mathbb{S}^{n-1}\right)$ построен ортонормированный базис $\left\{\psi_{j}\right\}_{j=1}^{\infty}$, такой, что $\int_{\mathbb{R}_{+}}\left(Z_{0}(t) \psi_{j}, \psi_{j}\right) \rho(t) d t=0$ для всех функций $\rho \in C_{0}^{\infty}((\tau, \infty))$. В этой ситуации теорема Лидского о совпадении матричного и спектрального следов влечет за собой равенство

$$
\sum_{j=1}^{\infty} \int_{\mathbb{R}_{+}}\left(Z_{V}(t) \psi_{j}, \psi_{j}\right) \rho(t) d t=\sum_{\lambda_{k} \in \Lambda} \int_{\mathbb{R}_{+}} e^{i \lambda_{k} t} \rho(t) d t,
$$

и ввиду леммы 2 справедливо следующее (см., например, [3])

ПРЕДЛОЖЕНИЕ 2. Регулярная часть обобщенной функиии $\Psi_{V}(t)=\operatorname{Tr}\left(U_{V}(t)\right.$ - $\left.U_{0}(t)\right)$ представима рядом Дирихле

$$
\Phi_{V}(t)=\sum_{\lambda_{k} \in \Lambda} e^{i \lambda_{k} t}, \quad t>0,
$$

где $\lambda_{k}$ упорядочены по неубыванию абсолютных величин.

\section{§4. Распределение и локализация резонансов}

Сопоставление формул (6) и (8) дает возможность делать заключения о структуре множества $\Lambda$. Так, например, из этого сопоставления следует (см. [3] и [17]), что если $c_{j}(V) \neq 0$ для некоторого $j \geqslant(n+1) / 2$, то множество $\Lambda$ бесконечно и, вследствие утверждения 1 , неограничено. Ниже будет показано, как можно на этом пути получить новую существенную информацию о расположении резонансов. Для простоты изложения рассматривается случай $n=3$.

Tеорема 2. Eсли $V(x) \in C_{0}^{\infty}\left(\mathbb{R}^{3}\right) u V(x) \not \equiv 0$, mo

$$
\sum_{\lambda_{k} \in \Lambda}\left|\lambda_{k}\right|^{-1 / 4}=\infty \text {. }
$$

ДокАЗАТЕЛЬСтво. Поскольку $\Lambda \subset\{\operatorname{Im} \lambda>0\}$ и $\left|\lambda_{k}\right| \rightarrow \infty, k \rightarrow \infty$, то для произвольного $N \in \mathbb{N}$ существует число $M \in \mathbb{N}$, такое, что

$$
\operatorname{Re}\left(\frac{e^{i \lambda_{k} t}}{i \lambda_{k}}\right)<0, \quad k=N+1, \ldots, N^{2}, t \in\left[0, \frac{1}{\left|\lambda_{M}\right|}\right] .
$$


Фиксируем последовательность пробных функций $\rho_{N} \in C_{0}^{\infty}\left(\mathbb{R}_{+}\right)$, удовлетворяющих следующим условиям:

$$
\rho_{N}(t)= \begin{cases}0 & \text { при } t \notin\left(\frac{1}{2\left|\lambda_{M}\right|}, \frac{2}{3\left|\lambda_{N}\right|}\right), \\ 1 \quad \text { при } t \in\left[\frac{1}{\left|\lambda_{M}\right|}, \frac{1,99}{3\left|\lambda_{N}\right|}\right],\end{cases}
$$

$\rho_{N}^{\prime}(t)>0$ при $t \in\left(\frac{1}{2\left|\lambda_{M}\right|}, \frac{1}{\left|\lambda_{M}\right|}\right)$ и $\rho_{N}^{\prime}(t)<0$ при $t \in\left(\frac{1,99}{3\left|\lambda_{N}\right|}, \frac{2}{3\left|\lambda_{N}\right|}\right)$; число $M=$ $M(N)$ выбрано так, что $\frac{1}{\left|\lambda_{M}\right|}<\frac{0,01}{3\left|\lambda_{N}\right|}$ и выполнено (9). Ввиду представления (8) обобщенная функция $\Psi_{V}(t)=\operatorname{Tr}\left(U_{V}(t)-U_{0}(t)\right)$ действует на пробную функцию $\rho_{N}$ по формуле

$$
\left\langle\Psi_{V}, \rho_{N}\right\rangle=\sum_{k \leqslant N}\left\langle e^{i \lambda_{k} t}, \rho_{N}\right\rangle+\sum_{k>N}\left\langle e^{i \lambda_{k} t}, \rho_{N}\right\rangle
$$

Согласно (7), имеем

$$
\left\langle\Psi_{V}, \rho_{N}\right\rangle=\int_{\mathbb{R}}\left(\frac{t}{16 \pi} \int V^{2} d x+r(t)\right) \rho_{N}(t) d t,
$$

где $r(t)=o(t)$ при $t \downarrow 0$, откуда непосредственно видно, что

$$
\left\langle\Psi_{V}, \rho_{N}\right\rangle=O\left(\left|\lambda_{N}\right|^{-2}\right), \quad N \rightarrow \infty .
$$

Оценим снизу вещественные части каждой из сумм в правой части равенства (10). Имеем

$$
\sum_{k \leqslant N}\left\langle e^{i \lambda_{k} t}, \rho_{N}\right\rangle=N \int_{\mathbb{R}} \rho_{N}(t) d t+i \sum_{k \leqslant N} \lambda_{k} \int_{\mathbb{R}} t \rho_{N}(t) d t+\sum_{k \leqslant N}\left\langle\alpha_{k}, \rho_{N}\right\rangle,
$$

где $\alpha_{k}(t)=e^{i \lambda_{k} t}-\left(1+i \lambda_{k} t\right)$ и

$$
\begin{aligned}
& \int_{\mathbb{R}} \rho_{N}(t) d t \geqslant \frac{1,98}{3\left|\lambda_{N}\right|}, \quad \int_{\mathbb{R}} t \rho_{N}(t) d t \leqslant \frac{2}{9\left|\lambda_{N}\right|^{2}}, \\
& \left|\left\langle\alpha_{k}, \rho_{N}\right\rangle\right| \leqslant \frac{9}{14}\left|\lambda_{k}\right|^{2} \int_{\mathbb{R}} t^{2} \rho_{N}(t) d t \leqslant \frac{4}{63} \frac{\left|\lambda_{k}\right|^{2}}{\left|\lambda_{N}\right|^{3}} .
\end{aligned}
$$

Таким образом, получается неравенство

$$
\begin{aligned}
\operatorname{Re}\left(\sum_{k \leqslant N}\left\langle e^{i \lambda_{k} t}, \rho_{N}\right\rangle\right) & \geqslant N \int_{\mathbb{R}} \rho_{N}(t) d t-\int_{\mathbb{R}} t \rho_{N}(t) d t \sum_{k \leqslant N}\left|\lambda_{k}\right|-\sum_{k \leqslant N}\left|\left\langle\alpha_{k}, \rho_{N}\right\rangle\right| \\
& \geqslant 0,36 \frac{N}{\left|\lambda_{N}\right|} .
\end{aligned}
$$

Вторую сумму в правой части (10) разобьем на два слагаемых:

$$
\sum_{k>N}\left\langle e^{i \lambda_{k} t}, \rho_{N}\right\rangle=\sum_{N+1}^{N^{2}}\left\langle e^{i \lambda_{k} t}, \rho_{N}\right\rangle+\sum_{k>N^{2}}\left\langle e^{i \lambda_{k} t}, \rho_{N}\right\rangle .
$$


Поскольку $\rho_{N}^{\prime}(t)>0$ при $t \in\left(\frac{1}{2\left|\lambda_{M}\right|}, \frac{1}{\left|\lambda_{M}\right|}\right)$, то в силу выбора $M$ справедливы неравенства

$$
\operatorname{Re}\left\{\frac{1}{i \lambda_{k}} \int_{1 / 2\left|\lambda_{M}\right|}^{1 /\left|\lambda_{M}\right|} e^{i \lambda_{k} t} \rho_{N}^{\prime}(t) d t\right\}<0, \quad k=N+1, \ldots, N^{2} .
$$

Замечая, что $\left|\int_{1,99 / 3\left|\lambda_{N}\right|}^{2 / 3\left|\lambda_{N}\right|} e^{i \lambda_{k} t} \rho_{N}^{\prime}(t) d t\right| \leqslant 1$, получаем

$$
\operatorname{Re} \sum_{N+1}^{N^{2}}\left\langle e^{i \lambda_{k} t}, \rho_{N}\right\rangle=-\operatorname{Re} \sum_{N+1}^{N^{2}} \frac{1}{i \lambda_{k}} \int_{\mathbb{R}} e^{i \lambda_{k} t} \rho_{N}^{\prime}(t) d t \geqslant-\sum_{N+1}^{N^{2}} \frac{1}{\left|\lambda_{k}\right|} .
$$

Далее, как нетрудно видеть, имеет место оценка

$$
\left|\sum_{k>N^{2}}\left\langle e^{i \lambda_{k} t}, \rho_{N}\right\rangle\right|=\left|\sum_{k>N^{2}} \frac{1}{i \lambda_{k}} \int_{\mathbb{R}} e^{i \lambda_{k} t} \rho_{N}^{\prime}(t) d t\right| \leqslant 2 \sum_{k>N^{2}} \frac{1}{\left|\lambda_{k}\right|},
$$

и, стало быть,

$$
\operatorname{Re} \sum_{k>N}\left\langle e^{i \lambda_{k} t}, \rho_{N}\right\rangle \geqslant-\left(\sum_{k>N} \frac{1}{\left|\lambda_{k}\right|}+\sum_{k>N^{2}} \frac{1}{\left|\lambda_{k}\right|}\right) .
$$

Допустим теперь, что ряд $\sum_{k=1}^{\infty}\left|\lambda_{k}\right|^{-1 / 4}$ сходится и, как следствие этого, $\left|\lambda_{N}\right|^{-1 / 4}=o(1 / N), N \rightarrow \infty$. В этом случае неравенство

$$
\sum_{k>N} \frac{1}{\left|\lambda_{k}\right|}+\sum_{k>N^{2}} \frac{1}{\left|\lambda_{k}\right|} \leqslant 0,35 \frac{N}{\left|\lambda_{N}\right|}
$$

выполнено для некоторой бесконечной последовательности $\left\{N_{s}\right\}$ значений $N$. В противном случае для достаточно больших $N$ имеем

$$
\frac{1}{\left|\lambda_{N}\right|}<\frac{1}{N^{4}}, \quad \sum_{k>N} \frac{1}{\left|\lambda_{k}\right|}+\sum_{k>N^{2}} \frac{1}{\left|\lambda_{k}\right|}>\frac{7}{20} \frac{N}{\left|\lambda_{N}\right|} .
$$

При этом справедлива оценка

$$
\sum_{k>N} \frac{1}{\left|\lambda_{k}\right|}+\sum_{k>N^{2}} \frac{1}{\left|\lambda_{k}\right|}<\int_{N}^{\infty} \frac{d x}{x^{4}}+\int_{N^{2}}^{\infty} \frac{d x}{x^{4}}=\frac{1}{3}\left(\frac{1}{N^{3}}+\frac{1}{N^{6}}\right)
$$

и, следовательно, $\left|\lambda_{N}\right|^{-1}<\frac{20}{21}\left(1+\frac{1}{N^{3}}\right) \frac{1}{N^{4}}$. Индуктивно повторяя проведенное рассуждение, получаем неравенство

$$
\left|\lambda_{N}\right|^{-1}<\left(\frac{20}{21}\right)^{m}\left(1+\frac{1}{N^{3}}\right)^{m} \frac{1}{N^{4}}
$$

с произвольным $m \in \mathbb{N}$. Это противоречит бесконечности множества $\Lambda=\left\{\lambda_{k}\right\}$ и, тем самым, доказывает существование искомой последовательности $\left\{N_{s}\right\}$.

Объединяя (12), (13) и (14), приходим к заключению, что в предположении сходимости ряда $\sum\left|\lambda_{k}\right|^{-1 / 4}$ имеет место неравенство

$$
\operatorname{Re}\left\langle\Psi_{V}, \rho_{N}\right\rangle \geqslant \frac{1}{100} \frac{N}{\left|\lambda_{N}\right|}, \quad N \in\left\{N_{s}\right\} .
$$

Ввиду неограниченности множества $\Lambda$ это, очевидно, несовместимо с оценкой $(11)$, и, таким образом, ряд $\sum\left|\lambda_{k}\right|^{-1 / 4}$ расходится. 
В ходе доказательства теоремы 2 было установлено, что $\left|\lambda_{N}\right|^{-1 / 4}$ не является величиной порядка $o(1 / N)$ при $N \rightarrow \infty$. Сопоставление этого факта с оценкой сверху для считающей функции количества резонансов позволяет обнаружить, что вне некоторой параболической окрестности мнимой оси находится бесконечно много резонансов.

СЛЕДСТВИЕ. Точки множества $\Lambda$, за исключением их конечного числа, не могут принадлежатъ области $\left\{|\operatorname{Re} \lambda|<(\operatorname{Im} \lambda)^{\gamma}\right\}, \gamma<1 / 12$.

ЗАмечАниЕ. Теорему 2 можно рассматривать как утверждение тауберова типа, позволяющее судить о расположении точек последовательности $\left\{\lambda_{k}\right\}$ по поведению суммы $\sum e^{i \lambda_{k} t}$ при $t \rightarrow 0$. Это может представлять интерес с точки зрения теории рядов Дирихле.

Автор благодарен рецензентам за конструктивные замечания к первоначальному варианту статьи.

\section{ЛитерАТУРА}

1. Лакс П., Филлипс Р. Теория рассеяния. Мир, М., 1972.

2. Вайнберг Б. Р. О собственных функциях оператора, отвечающих полюсам аналитического продолжения резольвенты через непрерывный спектр. Матем. сб., 87, № 2, 293-308 (1972).

3. Melrose R. Geometric scattering theory. Cambridge University Press, 1995.

4. Лидский В. Б. Несамосопряженные операторы, имеющие след. ДАН СССР, 125, №3, 485-488 (1959).

5. Арсенъев A. А. Сингулярные потенциалы и резонансы. Изд-во МГУ, М., 1974.

6. Базв А. И., Зельдович Я. Б., Переломов А. М. Рассеяние, реакции и распады в нерелятивистской квантовой механике. Наука, М., 1971.

7. Буслаев B. С. Формулы следов для оператора Шрёдингера в трехмерном пространстве. ДАН СССР, 143, №5, 1067-1070 (1962).

8. Павлов Б. С. Спектральный анализ диссипативного оператора Шрёдингера в терминах функциональной модели. В сб.: Итоги науки и техники. Современные проблемы математики. Фундаментальные направления, т. 65, ВИНИТИ, М., 1991, c. $95-163$.

9. Sjöstrand J. A trace formula and review of some estimates for resonances. In: Microlocal analysis and spectral theory, Math. Phys. Sci., Vol. 490, Kluwer Acad. Publ., 1997, pp. 377-437.

10. Zworski M. Quantum resonances and partial differential equations, Proc. ICM 2002, Vol. 3, pp. 243-252.

11. де Альфаро B., Редже Т. Потенциальное рассеяние. Мир, М., 1966.

12. Степин C. A. Волновые операторы для линеаризованного уравнения Больцмана в односкоростной теории переноса. Матем. сб., 192, №1, 139-160 (2001).

13. Bardos C., Guillot J. C., Ralston J. La relation de Poisson pour l'equation des ondes dans un ouvert non borne. Application a la theorie de la diffusion. Comm. Partial Differential Equations, 7, No. 8, 905-958 (1982).

14. Хёрмандер Л. Анализ линейных дифференциальных операторов с частными производными. Т. 3, Мир, М., 1987.

15. Бабич B. М. Метод Адамара и асимптотика спектральной функции дифференциального оператора второго порядка. Матем. заметки, 28, вып. 5, 689-694 (1980).

16. Melrose R. Scattering theory and the trace of the wave group. J. Funct. Anal., 45, 29-40 (1982).

17. Sá Barreto A., Zworski M. Existence of resonances in potential scattering. Comm. Pure Appl. Math., 49, 1271-1280 (1996).

Московский государственный университет, механико-математический факультет 